Quim. Nova, Vol. 31, No. 1, 84-88, 2008

\title{
MODIFICAÇÃO QUÍMICA E FÍSICA DO AMIDO DE QUIRERA DE ARROZ PARA APROVEITAMENTO NA INDÚSTRIA DE ALIMENTOS
}

\author{
Valéria Maria Limberger, Leila Picolli da Silva*, Tatiana Emanuelli, Carine Gláucia Comarela e Luciana Dalpieve \\ Patias \\ Departamento de Tecnologia e Ciência dos Alimentos, Centro de Ciências Rurais, Universidade Federal de Santa Maria, \\ Campus Universitário, 97105-900 Santa Maria - RS, Brasil
}

Recebido em 16/1/07; aceito em 14/6/07; publicado na web em 3/12/07

\begin{abstract}
CHEMICAL AND PHYSICAL MODIFICATION OF BROKEN RICE STARCH (Oryza sativa L.) FOR USE IN FOOD INDUSTRY. The study evaluated the efficiency of chemical (phosphorylation) and physical (extrusion) modifications of the starch of broken rice. Results demonstrated a reduction in the moisture content of extruded and phosphorylated broken rice and an increase in the ash content of phosphorylated broken rice. Both phosphorylation and extrusion increased cold water binding capacity, swelling power, and solubility. Extruded and phosphorylated pastes were stable under refrigeration, but only extruded paste was stable when submitted to freezing. Phosphorylated paste had the lowest viscosity and the highest stability during heating, while the extruded one gelatinized without heating, but had higher losses during heating.
\end{abstract}

Keywords: extrusion; phosphorylation; rheological characteristics.

\section{INTRODUÇÃO}

O arroz é alimento predominante na dieta da população de muitos países ${ }^{1}$, sendo o Brasil o $7^{\circ}$ maior produtor mundial deste cereal ${ }^{2}$. No beneficiamento do arroz branco são produzidos em média $14 \%$ de grãos quebrados, classificados como quirera. Este fato é um problema econômico para a indústria arrozeira porque o valor da quirera representa apenas a quinta parte daquele obtido na comercialização do grão inteiro. Porém, este subproduto é uma fonte rica em amido (aproximadamente $88 \%)^{3,4}$.

$\mathrm{O}$ amido é o mais abundante carboidrato de reserva em plantas, constituindo-se também em uma importante fonte energética para a alimentação humana. Amilose e amilopectina são os dois componentes macromoleculares dos grânulos de amido ${ }^{5,6}$. Além da sua importância nutricional, o amido apresenta importante papel tecnológico em alimentos processados ${ }^{5,-9}$. Na forma nativa o seu uso é mais restrito devido a algumas propriedades indesejáveis, tais como: insolubilidade em água fria, instabilidade frente a ciclos de congelamento e descongelamento e tendência a retrogradação ${ }^{10}$. Porém, estas podem ser melhoradas através de modificações físicas (extrusão) ou químicas (fosfatação) do amido ${ }^{11-15}$.

Entre as modificações químicas, destaca-se a fosfatação, a qual pode aumentar o poder de inchamento e a solubilização dos grânulos de amido e reduzir a retrogradação ${ }^{16,17}$. Em contra partida, a modificação física por extrusão tem sido explorada como processo alternativo às modificações químicas tradicionais, as quais são usualmente desenvolvidas em meio aquoso e geram grande volume de resíduos de difícil reciclagem ${ }^{18-20}$. Esta tecnologia tem se mostrado vantajosa por ser versátil e eficiente, de baixo custo, alta produtividade, curto tempo de reação e ausência de geração de resíduos ${ }^{21}$.

Neste contexto, visando a utilização e a agregação de valor à quirera de arroz, este trabalho foi conduzido com o objetivo de modificar física (extrusão) e quimicamente (fosfatação) o amido de quirera de arroz, avaliando a eficiência destas modificações através das características reológicas (capacidade de fixação de água

*e-mail:leilasliva@yahoo.com.br fria, poder de inchamento, solubilidade, viscosidade e sinérese) do amido nativo e modificado.

\section{PARTE EXPERIMENTAL}

\section{Material experimental}

A quirera de arroz foi obtida por doação de dois moinhos de Santa Maria - RS (Berleze e Cia Ltda e Favarin e Cia). As amostras utilizadas caracterizaram-se por ausência de sujidades e pontos pretos. A composição centesimal da amostra utilizada foi de $8,13 \%$ de proteína bruta; $22,5 \%$ de amilose; $1,15 \%$ de extrato etéreo; $0,45 \%$ de cinzas; $76,35 \%$ de amido total e $2,13 \%$ de fibra total.

\section{Extrusão}

A extrusão foi realizada na empresa "OK Alimentos" (Ijuí-RS), utilizando equipamento extrusor de alta compressão, dotado de rosca simples, com cilindro e rosca sem-fim curtos, operando a temperatura de $112 \pm 1{ }^{\circ} \mathrm{C}$ e rotação de $480 \mathrm{rpm}$.

A quirera de arroz foi condicionada a teor de umidade de $14 \%$ e introduzida na câmara de extrusão. Na seqüência, foi aplicada energia, gerada pela rotação e temperatura, levando à conversão do material sólido ao estado de massa pastosa, a fim de gelatinizar o amido. A temperatura da massa na câmara de extrusão imediatamente após a passagem pelos orifícios foi de $112 \pm 1{ }^{\circ} \mathrm{C}$, retirando a água existente no material e expandindo-o à medida que o mesmo atravessava os orifícios de saída. O tamanho e a forma foram determinados pela abertura do molde final $(3 \mathrm{~mm})$ e pela velocidade do sistema de corte, resultando em pellets de aproximadamente $3,5 \mathrm{~cm}$ de comprimento por $1 \mathrm{~cm}$ de diâmetro. Após a extrusão, a amostra passou por um secador rotatório de cilindro levemente inclinado, para reduzir a umidade do produto em até $5 \%$.

A amostra extrusada foi pulverizada e armazenada em recipiente hermeticamente fechado, sob refrigeração, até a realização das análises bromatológicas e reológicas. 


\section{Fosfatação}

O processo de fosfatação foi desenvolvido conforme descrito por Paschall ${ }^{22}$, com modificações: $100 \mathrm{~g}$ de quirera foram adicionadas em $167 \mathrm{~mL}$ de solução contendo 7,54\% de tripolifosfato de sódio $\left(\mathrm{Na}_{5} \mathrm{P}_{3} \mathrm{O}_{10}\right)$, agitada por $30 \mathrm{~min}$ em agitador mecânico e centrifugada por $5 \mathrm{~min}$ em $2000 \mathrm{rpm}$. O sedimento foi seco em estufa de ar forçado por $48 \mathrm{~h}$ a $45 \pm 2{ }^{\circ} \mathrm{C}$. A amostra seca foi pulverizada e colocada em estufa a $65 \pm 2{ }^{\circ} \mathrm{C}$ por $90 \mathrm{~min}$. Em seguida, foi transferida para uma estufa estacionária a $155 \pm 2{ }^{\circ} \mathrm{C}$ por 40 min. Após o resfriamento, foram adicionados $300 \mathrm{~mL}$ de etanol $50 \%$ e a amostra foi centrifugada durante $5 \mathrm{~min}$ a $2000 \mathrm{rpm}$, sendo a operação repetida mais duas vezes. $\mathrm{O}$ produto resultante foi seco em estufa de ar forçado a $45 \pm 2{ }^{\circ} \mathrm{C}$ por $48 \mathrm{~h}$. Para remover o excesso de sais de fósforo a amostra foi submetida à diálise $\mathrm{e}^{10,22}$. A quirera de arroz fosfatada foi armazenada em recipiente hermeticamente fechado, sob refrigeração.

\section{Análises bromatológicas}

Umidade, cinzas, extrato etéreo e proteína bruta foram determinados de acordo com as Normas Analíticas do Instituto Adolfo Lutz $^{23}$. Fibra alimentar total foi determinada segundo a metodologia número 991.43, recomendada pela $\mathrm{AOAC}^{24}$. A determinação de amido foi feita seguindo a técnica 996.11 da $\mathrm{AOAC}^{24}$ modificada por Walter ${ }^{25}$. A determinação de amilose foi realizada por análise iodométrica "Blue Value"26.

\section{Fósforo}

O teor de fósforo foi determinado de acordo com a técnica descrita por Tedesco e colaboradores ${ }^{27}$. Para se obter o grau de substituição da modificação, tomou-se o valor do fósforo ligado e usou-se a fórmula:

Grau de substituição = 162 P/(3100-124 P)

onde $\mathrm{P}=$ conteúdo de fósforo $(\%)$.

\section{Características reológicas}

A capacidade de ligação de água a frio (CLAF) foi realizada segundo método descrito por Medcalf e Gilles ${ }^{28}$. Nesta medida a amostra foi suspensa em água destilada em temperatura ambiente por 60 min, sendo a quantidade de água ligada na amostra determinada por pesagem do sedimento obtido após centrifugação.

$\mathrm{O}$ poder de inchamento (PI) e a solubilidade foram determinados segundo Schoch $^{29}$. Para obtenção destas medidas a amostra foi suspensa em água destilada, aquecida a $85^{\circ} \mathrm{C}$ por $30 \mathrm{~min}$. O poder de inchamento foi determinado pela quantidade de água retida na amostra, por pesagem do sedimento obtido após centrifugação. A solubilidade foi determinada pela quantidade de sólidos presentes no sobrenadante obtida por secagem.

A sinérese das pastas foi feita segundo Biliaderis ${ }^{30}$, sob refrigeração $\left(5^{\circ} \mathrm{C}\right.$ durante 14 dias $)$ e sob congelamento $\left(-18{ }^{\circ} \mathrm{C}\right.$ durante 28 dias). A viscosidade foi determinada por Analisador Rápido de Viscosidade (RVA), seguindo o protocolo padrão 2, descrito no método geral de empaste da "Newport Scientific Methods" (manual do fabricante) $)^{31}$.

\section{Delineamento experimental e análise estatística}

O experimento foi conduzido em delineamento completamente casualizado e os dados foram submetidos à análise de variância (ANOVA) e comparados por F-teste. Nos casos em que a análise de variância foi significativa, realizou-se o teste de Tukey a 5\% de probabilidade $(\mathrm{p}<0,05)$.

\section{RESULTADOS E DISCUSSÃO}

\section{Teor de umidade e cinzas}

Os resultados demonstram que tanto o processo de modificação por fosfatação quanto por extrusão provocaram reduções significativas no teor de umidade (Tabela 1), o que pode ser atribuído à alta temperatura aplicada nos processos.

A quirera de arroz fosfatada apresentou aumento de 11,2 vezes no teor de cinzas quando comparada à quirera nativa (Tabela 1), o que ocorreu devido à incorporação de grupos fosfato nas cadeias de amido $^{32}$. Sitohy e colaboradores ${ }^{10}$ também observaram aumento significativo no teor de cinzas do amido de arroz isolado nativo $(0,32 \%)$ para o fosfatado com grau de substituição de 0,07 (1,26\%), utilizando o mesmo princípio de técnica de fosfatação. $\mathrm{O}$ aumento no teor de cinzas não foi significativo na quirera de arroz modificada por extrusão.

Tabela 1. Umidade e cinzas (base seca) da quirera de arroz nativa e modificada

\begin{tabular}{lcc}
\hline Quirera & $\begin{array}{c}\text { Umidade } \\
(\%)\end{array}$ & $\begin{array}{c}\text { Cinzas } \\
(\%)\end{array}$ \\
\hline Nativa & $11,47 \pm 0,34^{\mathrm{a}}$ & $0,54 \pm 0,02^{\mathrm{b}}$ \\
Fosfatada & $8,06 \pm 0,05^{\mathrm{b}}$ & $6,08 \pm 0,02^{\mathrm{a}}$ \\
Extrusada & $8,10 \pm 0,07^{\mathrm{b}}$ & $0,62 \pm 0,05^{\mathrm{b}}$ \\
\hline
\end{tabular}

Valores seguidos de letras distintas na coluna diferem significativamente pelo teste de Tukey $(\mathrm{p}<0,05)$; os resultados são expressos como média \pm desvio padrão.

\section{Teor de fósforo}

O processo de diálise reduziu significativamente o conteúdo de fósforo das amostras (Tabela 2). Mesmo após a diálise, o teor de fósforo das amostras submetidas à fosfatação foi significativamente superior à nativa, indicando que o processo foi efetivo para promover a ligação do fósforo ao amido. A quirera de arroz dialisada apresentou teor de fósforo semelhante ao relatado por Paschall ${ }^{22}$ para esta técnica (G.S. 0,02), ficando dentro do limite recomendado para uso em alimentos pelo Codex Alimentarius ( $0,4 \%$ de fósforo para amido de arroz) $)^{33}$.

\section{Capacidade de ligação à água a frio (CLAF)}

A fosfatação aumentou significativamente a capacidade de liga-

Tabela 2. Conteúdo de fósforo e grau de substituição da quirera de arroz nativa e modificada, dialisadas e não dialisadas

\begin{tabular}{lccc}
\hline Quirera & $\begin{array}{c}\text { Fósforo em } \\
\text { amostras não } \\
\text { dialisadas } \\
(\%)\end{array}$ & $\begin{array}{c}\text { Fósforo em } \\
\text { amostras } \\
\text { dialisadas } \\
(\%)\end{array}$ & $\begin{array}{c}\text { Grau de } \\
\text { substituição } \\
\text { (dialisada) }\end{array}$ \\
\hline Nativa & $0,1043 \pm 0,006^{\mathrm{b}} \mathrm{A}$ & $0,06320 \pm 003^{\mathrm{b}} \mathrm{B}$ & $0,003 \pm 0,0003$ \\
Fosfatada & $0,9151 \pm 0,015^{\mathrm{a}} \mathrm{A}$ & $0,31050 \pm 004^{\mathrm{a}} \mathrm{B}$ & $0,024 \pm 0,0020$
\end{tabular}

Valores seguidos de letras distintas, minúsculas na coluna e maiúsculas na linha, diferem significativamente pelo teste de Tukey $(\mathrm{p}<0,05)$; os resultados são expressos como média \pm desvio padrão. 
ção da água a frio da quirera de arroz (Tabela 3), o que ocorre devido à repulsão entre grupos fosfato ligados às moléculas de amido no interior dos grânulos. Esses grupos possuem cargas negativas que se repelem, facilitando a penetração e absorção da água ${ }^{34}$, sendo que a força do gel será dependente da capacidade de formação de pontes de hidrogênio entre moléculas de amido e água, bem como da retenção física de moléculas de água no interior do grânulo de amido ${ }^{5}$. No entanto, o aumento do valor de CLAF foi mais pronunciado quando aplicado o processo de extrusão, o que é explicado pela grande alteração física que este promove na estrutura granular e molecular do amido, sendo mais efetivo na absorção e retenção da água a frio quando comparado ao processo de fosfatação.

Tabela 3. Capacidade de ligação de água a frio da quirera de arroz nativa e modificada

\begin{tabular}{lc}
\hline Quirera & Capacidade de ligação de água a frio $(\%)$ \\
\hline Nativa & $112,51 \pm 1,46^{\mathrm{c}}$ \\
Fosfatada & $191,06 \pm 2,54^{\mathrm{b}}$ \\
Extrusada & $480,34 \pm 1,66^{\mathrm{a}}$
\end{tabular}

Valores seguidos de letras distintas na coluna diferem significativamente pelo teste de Tukey $(\mathrm{p}<0,05)$; os valores são expressos como média \pm desvio padrão.

O valor encontrado para CLAF neste estudo para a amostra extrusada (Tabela 3) está de acordo com Bryant e colaboradores ${ }^{35}$, que observaram $448 \%$ de absorção de água em farinhas de arroz extrusado de grão longo, em condições de extrusão semelhantes. $\mathrm{O}$ conteúdo de umidade, agindo como um plastificante durante a extrusão, reduz a degradação dos grânulos de amido e isso resulta em um aumento da capacidade de absorção ${ }^{1}$. Em uma lâmina padrão (rosca a baixa velocidade) e/ou baixa temperatura, pode-se esperar mais cadeias de polímeros não danificadas e uma grande disponibilidade de grupos hidrofílicos, os quais podem reter mais água, resultando em valores de absorção mais altos ${ }^{36}$.

\section{Poder de inchamento e solubilidade}

O poder de inchamento e a solubilidade aumentaram significativamente na quirera de arroz fosfatada (Tabela 4). Este resultado condiz com os dados de Sitohy e colaboradores ${ }^{10}$, que mostraram altos valores de solubilidade em água para o amido de arroz fosfatado com baixo grau de substituição. Segundo estes autores, a introdução de grupos fosfato nos grânulos de amido pode reduzir as forças inter-moleculares de ligação, devido à repulsão dos grupos fosfato negativamente carregados nas moléculas de amido, aumentando o poder de inchamento e a solubilidade.

A determinação do poder de inchamento e solubilidade ocorre em temperatura elevada, promovendo a quebra de pontes de hidrogênio; as moléculas de água fixam-se deixando livres grupos hidroxila, e os grânulos continuam a inchar, resultando no aumento da solubilidade do amido ${ }^{32}$.

A extrusão também aumentou o poder de inchamento e solubilidade quando comparada à amostra não modificada; porém, resultou em um menor poder de inchamento quando comparada à fosfatada, o que pode estar relacionado à degradação do amido, que causa uma redução na capacidade de retenção de água das moléculas frente ao aquecimento, como resultado do decréscimo no tamanho molecular ${ }^{37}$. A solubilidade da quirera extrusada também se mostrou menor neste estudo quando comparada à fosfatada. Esta é relacionada com a presença de moléculas solúveis produzidas na dextrinização, o que pode ser usado como um indicador do grau de dano molecular ${ }^{38}$.

Bryant e colaboradores ${ }^{35}$ determinaram índice de solubilidade em água para farinha de arroz extrusada e encontraram valor semelhante $(37,86 \%)$ ao deste estudo. Wen e colaboradores ${ }^{39}$ indicaram que a velocidade de rotação da rosca extrusora tem efeito direto na distribuição do tamanho dos polissacarídeos, sendo que quanto mais alta, maior a fragmentação. Adicionalmente, Guha e Bhattacharya ${ }^{37}$ demonstraram o efeito da temperatura do barril de extrusão sobre a solubilidade, sendo que amostras extrusadas a altas temperaturas e velocidade de rotação demonstraram maior solubilidade.

Tabela 4. Poder de inchamento e solubilidade da quirera de arroz nativa e modificada

\begin{tabular}{lcc}
\hline Quirera & $\begin{array}{c}\text { Poder de } \\
\text { inchamento* } \\
\text { (vezes) }\end{array}$ & $\begin{array}{c}\text { Solubilidade* } \\
(\%)\end{array}$ \\
\hline Nativa & $9,44 \pm 0,58^{\mathrm{c}}$ & $18,17 \pm 0,83^{\mathrm{c}}$ \\
Fosfatada & $20,19 \pm 0,56^{\mathrm{a}}$ & $47,06 \pm 0,63^{\mathrm{a}}$ \\
Extrusada & $14,3 \pm 0,06^{\mathrm{b}}$ & $35,91 \pm 0,46^{\mathrm{b}}$ \\
\hline
\end{tabular}

Valores seguidos de letras distintas na coluna diferem significativamente pelo teste de Tukey $(\mathrm{p}<0,05)$; os resultados são expressos como média \pm desvio padrão.

\section{Sinérese}

A sinérese foi significativamente influenciada pela modificação, bem como pelo tipo de modificação (Figura 1), em amostras mantidas por 14 dias sob refrigeração $\left(5^{\circ} \mathrm{C}\right)$. A quirera fosfatada teve perda de água significativamente reduzida em relação à nativa. Este fato pode ser explicado porque a incorporação de fosfato no amido previne a retrogradação, responsável pela perda de água por sinérese, pois evita um contato maior entre as moléculas de amilose que se solubilizam durante o aquecimento e saem do interior do grânulo, impedindo a formação de microcristais ${ }^{40}$. A redução da sinérese na quirera de arroz modificada por fosfatação ainda pode ser explicada pela repulsão entre os grupos de ésteres de fosfato, que impede a ligação das moléculas de hidrogênio do amido ${ }^{41,42}$.

A quirera extrusada apresentou maior estabilidade, indicando maior resistência à retrogradação. Isto pode ser consequiência da alteração estrutural promovida pela extrusão que, devido ao efeito de quebra da estrutura, promove fragmentação das moléculas de amilose em tamanhos muito pequenos para formar a estrutura de dupla hélice característica, que favorece a retrogradação ${ }^{15}$.

Durante a armazengem sob congelamento $\left(-18{ }^{\circ} \mathrm{C}\right.$ durante 28

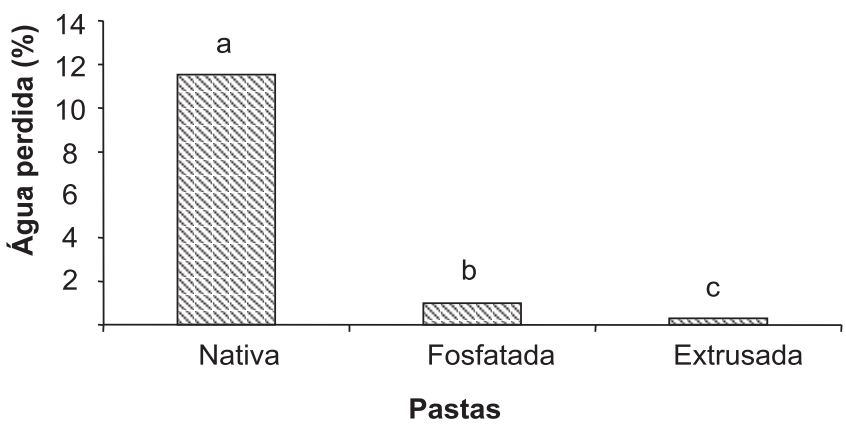

Figura 1. Sinérese das pastas (10\%) de quirera de arroz nativa e modificada durante armazenagem a $5{ }^{\circ} \mathrm{C}$ por 14 dias. As barras que apresentam letras distintas diferem significativamente entre si pelo teste de Tukey $(p<0,05)$ 
Tabela 5. Propriedades de viscosidade da quirera de arroz nativa, fosfatada e extrusada

\begin{tabular}{|c|c|c|c|c|c|}
\hline \multirow[t]{2}{*}{ Amostra } & $\begin{array}{c}\text { Viscosidade } \\
\text { no Pico }\end{array}$ & "Breakdown" & $\begin{array}{c}\text { Viscosidade } \\
\text { Final }\end{array}$ & "Setback" & \multirow{2}{*}{$\begin{array}{c}\text { Tempo } \\
\text { de Pico } \\
(\min )\end{array}$} \\
\hline & \multicolumn{4}{|c|}{ RVU } & \\
\hline Nativa & $247,3 \pm 47,0^{\mathrm{b}}$ & $56,05 \pm 33,50^{\mathrm{b}}$ & $420,9 \pm 39,3^{a}$ & $229,6 \pm 418,2^{\mathrm{a}}$ & $9,1 \pm 0,0^{\mathrm{b}}$ \\
\hline Fosfatada & $64,7 \pm 3,3^{c}$ & $0,14 \pm 0,10^{\mathrm{c}}$ & $113,6 \pm 7,1^{\mathrm{c}}$ & $49,3 \pm 3,7^{\mathrm{c}}$ & $12,9 \pm 0,2^{\mathrm{a}}$ \\
\hline Extrusada & $73,6 \pm 5,2^{\mathrm{c}}$ & $65,39 \pm 4,70^{\mathrm{b}}$ & $16,0 \pm 1,6^{\mathrm{d}}$ & $7,72 \pm 0,8^{\mathrm{d}}$ & $4,4 \pm 2,2^{\mathrm{c}}$ \\
\hline
\end{tabular}

Valores seguidos de letras distintas na coluna diferem significativamente pelo teste de Tukey ( $<<0,05)$; os resultados são expressos como média \pm desvio padrão. RVU: unidade do analisador rápido de viscosidade.

dias), também foi observado o efeito do tipo de modificação sobre a perda de água por sinérese (Figura 2), a qual não diferiu significativamente entre as amostras fosfatada e nativa. $\mathrm{O}$ mesmo não ocorreu com a quirera de arroz modificada por extrusão, que apresentou sinérese significativamente menor que as demais, indicando que a alteração conformacional na estrutura do amido foi intensa e estável o suficiente para reduzir o processo de retrogradação também durante o congelamento.

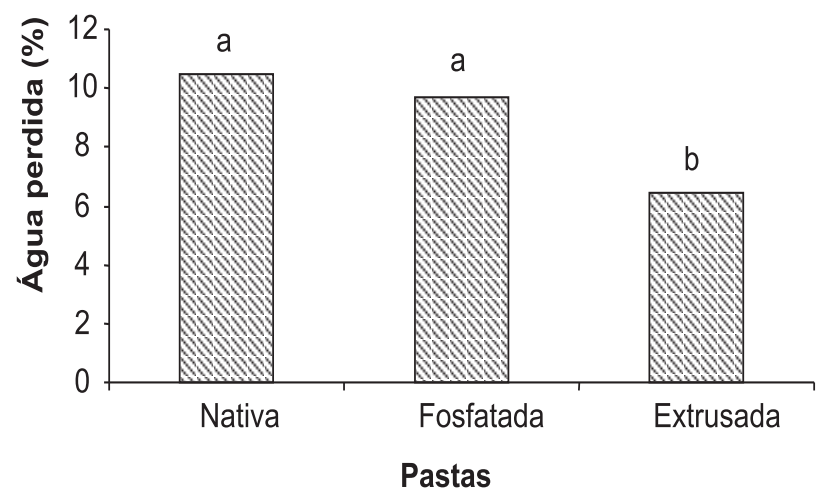

Figura 2. Sinérese das pastas (10\%) de quirera de arroz nativa e modificada durante armazenagem a $-18^{\circ} \mathrm{C}$ por 28 dias. As barras que apresentam letras distintas diferem significativamente entre si pelo teste de Tukey $(p<0,05)$

\section{Viscosidade}

A viscosidade no pico de empaste da quirera de arroz fosfatada foi significativamente menor que a viscosidade da quirera de arroz nativa (Tabela 5), o que pode ser explicado pelas condições a que a quirera de arroz foi submetida durante a fosfatação, como a concentração de fósforo na mistura, tempo e alta temperatura de fosfatação, o que leva à degradação do produto ou à formação de ligações cruzadas, sendo que estes dois fatores diminuem a viscosidade ${ }^{17}$.

A quirera de arroz extrusada também demonstrou significativa redução na viscosidade (Tabela 5). Isto indica forças de ligação fracas e uniformes ${ }^{42}$, conseqüência da degradação (dextrinização) do amido durante o processo de extrusão $0^{43}$. As curvas de viscosidade dos produtos extrusados mostradas por Ilo e colaboradores ${ }^{45} \mathrm{e}$ Hagenimana e colaboradores ${ }^{1}$ também exibiram viscosidade menor comparada às farinhas não extrusadas.

A quirera de arroz fosfatada mostrou-se mais estável frente ao aquecimento, apresentando quebra ("breakdown") reduzida quando comparada com a nativa, que mostrou elevado pico de viscosidade. Os amidos que são capazes de inchar a um alto grau também são menos resistentes à quebra no cozimento exibindo, conseqüentemente, diminuição significante na viscosidade depois de atingir o valor máximo ${ }^{44}$.

A quirera de arroz extrusada formou pasta em temperatura ambiente, mas apresentou valor de "breakdown" elevado. O comportamento da curva de empaste desta amostra assemelha-se ao encontrado por Bryant ${ }^{35}$ para farinha de arroz extrusada em condições semelhantes.

O "setback" reflete o grau de retrogradação da pasta de amido, sendo que quanto menor o "setback" menor a retrogradação e, conseqüentemente, a sinérese, o que foi claramente observado neste estudo, quando confrontamos os resultados das Figuras 1 e 2 com os valores de "setback" observados na Tabela 5 .

\section{CONCLUSÕES}

Tanto a fosfatação (GS 0,024) quanto a extrusão modificaram as características reológicas da quirera de arroz nativa, o que amplia a utilização deste subproduto na indústria de alimentos. No entanto, a modificação mais indicada dependerá da aplicação pretendida, sendo que a extrusão parece ser mais adequada a produtos que dispensem aquecimento no seu preparo. Por sua vez, a fosfatação é alternativa na utilização de aquecimento. No entanto, devese considerar que a extrusão não utiliza reagente químico, evitando a geração de resíduos; concomitantemente, o produto obtido não possui limite de uso estipulado pela legislação, ao contrário daquele obtido por fosfatação.

\section{AGRADECIMENTOS}

Ao apoio financeiro da CAPES e do Instituto Rio Grandense do Arroz (IRGA), e à Berleze e Cia Ltda e Favarin e Cia pelo fornecimento das amostras de quirera. L. P. Silva é bolsista PRODOCCAPES e T. Emanuelli é bolsista de produtividade em pesquisa do CNPq (proc. 304257/2004-4).

\section{REFERÊNCIAS}

1. Hagenimana, A.; Ding, X.; Fang, T.; J. Cereal Sci. 2005, 43, 38.

2. http://www.conab.gov.br, acessada em Dezembro 2005.

3. Coffman, W. R.; Juliano, B. O. Em Nutritional quality of cereal grains: Genetic and agronomic improvement; Olson, R. A.; Frey, K. J., eds.; American Society of Agronomy: Madison, 1987, cap. 5.

4. Silva, L. P.; Nörnberg, J. L.; Emanuelli, T.; Fagundes, C. A. A.; Ortolan, F.; Denardin, C. C.; Resumos da 3rd International Temperate Rice Conference, Punta Del Este, Uruguay, 2003.

5. Sandhu, K. S.; Singh, N.; Kaur, N.; J. Food Eng. 2004, 64, 119.

6. Vandeputte, G. E.; Vermeylen, R.; Geeroms, J.; Delcour, J. A.; J. Cereal Sci. 2003, 38, 43.

7. Barreto, P. L. M.; Beirão, L. H.; Ciênc. Tecnol. Aliment. 1999, 19, 45

8. Hermansson, A. M.; Svegmark, K.; Trends Food Sci. Technol. 1996, 7, 345.

9. Kusunose, C.; Fujjii, T.; Matsumoto, H.; Cereal Chem. 1999, 76, 920.

10. Sitohy, M. Z.; El-Saadany, S. S.; Labib, S. M.; Ramadan, M. F.; Starch/ Staerke 2000, 52, 101.

11. Alves, R. M. L.; Grossmann, M. V. E.; Silva, R. S. S. F.; Food Chem. 1999, $67,123$. 
12. Branen, A. L.; Davidson, P. M.; Salminen, S.; Food additives, Series Food Sci. Technol., Marcel Dekker, Inc.: New York, 1990.

13. Eliasson, A. C.; Gudmundsson, M.; Starch: physicochemical and functional aspects. Carbohyd in Food, Marcel Dekker, Inc.: New York, 1996, p. 431.

14. Kim, H. R.; Hermanson, A. M.; Eriksson, C. E.; Starch/Staerke 1992, 42, 418.

15. Schirmer, M. A.; Toledo, M. C. F.; Reys, F. G. R.; Bol. SBCTA 1986, 20, 55.

16. Sivak, M. N.; Preiss, J.; Adv. Food Nutr. Res. 1998, 41, 163.

17. Schirmer, M. A.; Toledo, M. C. F.; Reys, F. G. R.; Bol. SBCTA 1986, 20, 153.

18. Chang, Y. H.; Lii, C. Y.; J. Food Sci. 1992, 57, 203.

19. Meuser, F.; Gimmler, N. Em Trends in Food Processing; Ghee, A. H., ed.; Singapore Institute of Science and Technology: Singapore, 1989, p. 289.

20. Salay, E.; Ciacco, C. F.; Starch/Staerke 1990, 42, 15

21. Nabeshima, E. H.; Grossmann, M. V. E.; Carbohydr. Polym. 2001, 45, 347.

22. Paschall, E. F. Em Methods in Carbohydrate Chemistry; Whiistler, R. L., ed.; Academic Press: New York, 1964, vol. IV, p. 294.

23. Normas Analíticas do Instituto Adolfo Lutz; Métodos Químicos e Físicos para Análise de Alimentos - 1, $3^{\mathrm{a}}$ ed., São Paulo, 1985.

24. AOAC; Official Methods of Analysis, 16 $6^{\text {th }}$ ed., Arlington, 1995.

25. Walter, M.; Dissertação de Mestrado, Universidade Federal de Santa Maria, Brasil, 2005.

26. Gilbert, G. A.; Spragg, S. P. Em ref. 22, p. 168

27. Tedesco, M. J.; Gianello, C.; Bohnen, H.; Análises de Solos, Plantas e outros Materiais, $2^{a}$ ed.; Departamento de Solos da UFRGS: Porto Alegre, 1995 , p. 174
28. Medcalf, D. G.; Gilles, K. A.; Cereal Chem. 1965, 42, 561

29. Schoch, T. J. Em Methods in Carbohydrate Chemistry; Whiistler, R. L. ed.; Academic Press: New York, 1964, vol. III, p. 107.

30. Biliaderis, C. G.; Agric. Food Chem. 1982, 30, 925.

31. Newport Scientific Method ST-00, Revision 3, November 1998; Developed by A. E. Staley Manufacturing Co., Decatur, IL 62525, USA, 1998.

32. Stahl, J. A.; Dissertação de Mestrado, Universidade Federal de Santa Maria, Santa Maria, Brasil, 2003.

33. http//:www.fao.org, acessada em Janeiro 2006

34. Sitohy, M. Z.; Labib, S. M.; El-Saadany, S. S.; Ramadan, M. F.; Starch/ Staerke 2000, 52, 95.

35. Bryant, R. J.; Kadan, R. S.; Champagne, E. T.; Vinyard, B. T.; Boykin, D.; Cereal Chem. 2001, 78, 131.

36. Gomez, M. H.; Aguilera, J. M.; J. Food Sci. 1983, 48, 378

37. Guha, M.; Ali, S. Z.; Bhattacharya, S.; J. Food Eng. 1997, 32, 251.

38. Paton, D.; Spratt, W. A.; Cereal Chem. 1981, 58, 216.

39. Wen, L. F.; Rodis, P.; Wasseman, B. P.; Cereal Chem. 1990, 67, 268.

40. Cruz, R.; El Dash, A. A. M.; Bol. SBCTA 1984, 18, 371.

41. Craig, S. A. S.; Maningat, C. C.; Seib, P. A.; Hoseney, R. C.; Cereal Chem. 1989, 66, 173

42. Leach, H. W.; McCowen, L. D.; Schoch, T. J.; Cereal Chem. 1959, 36, 534

43. Ilo, S.; Liu, Y.; Berghofer, E.; Lebensm Wiss Technol. 1999, 32, 79.

44. Singh, N.; Singh, J.; Kaur, L.; Sodhi, N. S.; Gill, B.; Food Chem. 2003, 81,219 